Georgian Mathematical Journal

Volume 11 (2004), Number 4, 645-654

\title{
EXTENSIONS WITH ABELIAN KERNELS IN PROTOMODULAR CATEGORIES
}

\author{
D. BOURN AND G. JANELIDZE
}

\begin{abstract}
As observed by J. Beck, and as we know from M. Barr's and his joint work on triple cohomology, the classical isomorphism Opext $\cong H^{2}$ that describes group extensions with abelian kernels, can be deduced from the equivalence between such extensions and torsors (in an appropriate sense). The same is known for many other "group-like" algebraic structures, and now we present a purely-categorical version of that equivalence, essentially by showing that all torsors are extensions with abelian kernels in any pointed protomodular category, and by giving a necessary and sufficient condition for the converse.
\end{abstract}

2000 Mathematics Subject Classification: 18G50, 18D35, 20J06, 18C10, $18 \mathrm{G} 60$.

Key words and phrases: Protomodular category, semi-abelian category, Opext functor, extension, torsor.

\section{INTRODUCTION}

The set $\operatorname{Ext}(B, K)$ of isomorphism classes of extensions of a group $B$ with an abelian kernel $K$ splits into disjoint union

$$
\operatorname{Ext}(B, K)=\bigsqcup_{\xi \in \Xi} \operatorname{Opext}(B, K, \xi),
$$

where $\Xi$ is the set of all $B$-module structures on $K$, and each $\operatorname{Opext}(B, K, \xi)$ has (a group structure and) a cohomological description

$$
\operatorname{Opext}(B, K, \xi) \cong H^{2}(B, K, \xi)
$$

(see S. Mac Lane [19] for details and references, where however the symbol "Ext" is used only for an abelian category). This and several other similar classical theories (for associative or Lie algebras and for some other "group-like" structures) have a unified treatment by G. Orzech [20], which was suggested by M. Barr and in fact is based on the fact that, in all categories of interest, we have:

The elements of $\operatorname{Op} \operatorname{pxt}(B, K, \xi)$ are the same as torsors over the semidirect product of $B$ and $(K, \xi)$, considered as an (abelian) group in $(\mathbf{C} \downarrow B)$, where $\mathbf{C}$ is the ground category; or, briefly, that extensions=torsors. It was J. Beck ${ }^{1}$ who first discovered this fact for groups and who realized, as also M. Barr explained

${ }^{1}$ J. Beck's Ph.D. Thesis (1967) is now available as [J. M. Beck, Triples, algebras and cohomology, Repr. Theory Appl. Categ. No. 2 (2003), 1-59; http://www.tac.mta.ca/tac/]. 
in his talk [1], that a $B$-module in a general category $\mathbf{C}$ is to be defined as an abelian group in $(\mathbf{C} \downarrow B)$.

Our aim in this paper is to make "extensions=torsors" a purely-categorical theorem, using the categorical notion of a semi-direct product introduced in [10]. In particular it follows that what M. Gerstenhaber [13] calls Baer extension theory is a special case of the general theory of torsors.

The main reason for "extensions=torsors", as one can see in Section 2 below, is that, for every split epimorphism, its kernel and its splitting are jointly epic; it is a consequence of Split Short Five Lemma, which in fact says that the category of split extensions of a fixed object with a fixed kernel is a groupoid, and which also plays a crucial role by itself. This suggests that the right categorical context for our constructions is the context of (pointed finitely complete) categories satisfying Split Short Five Lemma, i.e., of (pointed) protomodular categories in the sense of [4] (see also [2]). The comparison with Gerstenhaber theory then suggests to ask, how far are his Moore categories from the protomodular ones?

The answer is a long story, which involves semi-abelian categories defined in [16] as pointed finitely complete and finitely complete protomodular categories that are Barr exact. The main theorem of [16] asserts that this list of conditions is equivalent to the list of Old Categorical Axioms, which essentially are all (first order) categorical conditions used in homological algebra and radical theory in fifties and sixties, that hold in any variety of groups with multiple operators in the sense of P. Higgins [14]. Gerstenhaber's definition of a Moore category (see also [24]) requires almost all these axioms, plus also a stronger one, which holds in many classical-algebraic categories, but unfortunately not in every variety of groups with multiple operators. We knew about this disagreement of definitions already in 1997 when we almost finished this paper. We, however, postponed publishing it, because, not having yet understood the connection with pregroupoid/commutator theory, we used more complicated arguments that we felt were to be simplified, and we did not know how to do that. This problem is solved now, and our present version is very simple:

Let $\mathbf{C}$ be a Mal'tsev category, $B$ an object in $\mathbf{C}$, and $G$ a group in $(\mathbf{C} \downarrow B)$. The equivalence between the category of all torsors over groupoids in $\mathbf{C}$ and the category of pregroupoids in $\mathbf{C}$ (due to A. Kock [17] and [18]) induces an embedding of the category of $G$-torsors in $(\mathbf{C} \downarrow B)$ into the category of extensions of $B$. This follows from the fact that any span in a Mal'tsev category has at most one pregroupoid structure (by commutator theory, but originally from $[12$, Proposition 3.5]). If, moreover, the category $\mathbf{C}$ is pointed protomodular, then the morphisms of torsors admit better description in terms of kernels of the corresponding extensions, and we arrive to the desired presentation of $\operatorname{Ext}_{\mathbf{C}}(B, K)$ as the disjoint union of all $\operatorname{Opext}_{\mathbf{C}}(B, K, \xi)$. We should also mention that we in fact need protomodularity (more precisely even the stronger property of the existence of semidirect products) once more in order to have a good definition of actions $\xi$ above.

According to this approach, torsors correspond to those extensions $\alpha: A \rightarrow$ $B=A / R$, for which the Pedicchio commutator $[R, R]$ is trivial, as in 1.1 below. 
Here $R$ is the equivalence relation that appears as the kernel pair of $\alpha$, and the abovementioned strong axiom used in the definition of a Moore category is exactly what makes the triviality of $[R, R]$ equivalent to the abelianness of the (usual) kernel of $\alpha$ (see [7]). Moreover, as follows from our results, it is the only reason why Gerstenhaber's theory needs that axiom.

Another thing is that since our ground category is not (required to be) semiabelian, and not even regular, but merely protomodular, our definition of an extension is different from the usual one in homological algebra: instead of asking its epi-part to be a cokernel of its kernel, i.e., a normal epimorphism, we are asking it to be an effective descent morphism. In the case of a semi-abelian category there is no difference of course.

Finally, we would like to point out the following: Although we assume here familiarity with some recent categorical-algebraic developments, the rest of the paper is in fact "more categorical" than this Introduction - in the sense that all new theorems we prove are first formulated in Section 2 in terms of categories of extensions (or torsors) rather than the corresponding set-valued functors Opext, as, say, in Mac Lane's book [19]. However we return to Opext functors in Section 3.

\section{Pregroupoids and Commutators in Mal'tsev Categories}

The notion of commutator of two congruences in a Mal'tsev variety was introduced by J. D. H. Smith [25], and then generalized in various ways by many authors. The first categorical definition, in the context of exact Mal'tsev categories with coequalizers, is due to M. C. Pedicchio [21], [22] (see also [23]). It was later extended to spans in a category with finite limits and arbitrary intersections of subobjects in [15] (which has first appeared as a Trieste University preprint in 1998), although [15] mainly deals with varieties of universal algebras. Commutator theory in arbitrary (non-exact) Mal'tsev categories was then systematically developed in [8], [9], [6], [7], using also the older results of [12]. We are interested here in the following special case which certainly agrees with all definitions ever used:

Definition and Theorem 1.1. Let $\alpha: A \rightarrow B$ be a morphism in a finitely complete Mal'tsev category $\mathbf{C}$, and $R=A \times_{B} A$ the corresponding equivalence relation on $A$ (=the kernel pair of $\alpha$ ). We say that the commutator $[R, R]$ is trivial, or that $R$ centralizes itself, if the following equivalent conditions hold:

(a) the span $B \stackrel{\alpha}{\longleftarrow} A \stackrel{\alpha}{\longrightarrow} B$ admits a pregroupoid structure (in the sense of A. Kock [18]);

(b) the morphism $\alpha: A \rightarrow B$, considered as an object in $(\mathbf{C} \downarrow B)$, admits a unique Mal'tsev operation, i.e., a ternary operation $p$ satisfying $p(x, y, y)=x=$ $p(y, y, x)$ (in the standard-algebraic notation);

(c) the morphism $\alpha: A \rightarrow B$, considered as an object in $(\mathbf{C} \downarrow B)$, admits an associative Mal'tsev operation, where the associativity means $p(x, y, p(z, u, \nu))=$ $x=p(p(x, y, z), u, \nu)$ in the notation above; 
(d) the morphism $\alpha: A \rightarrow B$, considered as an object in $(\mathbf{C} \downarrow B)$, is abelian in the sense of commutator theory, i.e., its largest commutator is trivial; here again, since $(\mathbf{C} \downarrow B)$ is a Mal'tsev category, all notions of (the triviality of) commutator agree.

Whenever $\mathbf{C}$ is pointed, the conditions above imply the abelianness of the kernel of $A \rightarrow B$, and the converse is true when $\mathbf{C}$ is strongly protomodular [7], which (together with Barr exactness) is essentially the same as to be a Moore category in the sense of M. Gerstenhaber [13] (see also Introduction). The precise relationship of these two notions is described in details by the first author's student D. Rodelo [24].

Let us also mention the following reformulation (essentially due to M. C. Pedicchio) of the equivalence $(\mathrm{b}) \Leftrightarrow(\mathrm{c})$ of 1.1 in the more general context of arbitrary spans:

Theorem 1.2. The forgetful functor from pregroupoids to spans in a finitely complete Mal'tsev category is full and faithful, and full on subobjects.

In order to distinguish pregroupoids with the underlying spans of the form

$B \stackrel{\alpha}{\leftarrow} A \stackrel{\alpha}{\longrightarrow} B$ from general ones, we will call them endo-pregroupoids; if in addition $A \rightarrow B$ was an effective descent morphism, then we will use the expression regular endo-pregroupoid. That is, "regular" has the same meaning as in [17] and [18].

\section{TORSORS AS EXTENSIONS}

Definition 2.1. An internal group action $h: G \times X \rightarrow X$ in a finitely complete category $\mathbf{C}$ is said to be a torsor, if it satisfies the following conditions:

(a) the morphism $\left\langle h, \operatorname{pr}_{2}\right\rangle: G \times X \rightarrow X \times X$ induced by $h$ and the second projection $G \times X \rightarrow X$ is an isomorphism;

(b) the morphism $X \rightarrow t$, where $t$ denotes a terminal object of $\mathbf{C}$, is an effective descent morphism.

When $G$ fixed, we will also say that $(X, h)$ is a $G$-torsor. We will need to consider the $G$-torsors in $(\mathbf{C} \downarrow B)$, for an arbitrary "base" object $B$ in $\mathbf{C}$ and an arbitrary group $G$ in $(\mathbf{C} \downarrow B)$. Putting all these torsors together, and defining appropriate morphisms between them using the fact that a group in $(\mathbf{C} \downarrow B)$ can always be considered as a groupoid in $\mathbf{C}$, we obtain a category, which we will denote by Tors $\mathbf{C}$. Specifically, a morphism from a torsor $h: G \times(A, \alpha) \rightarrow(A, \alpha)$ in $(\mathbf{C} \downarrow B)$ to a torsor $h^{\prime}: G^{\prime} \times\left(A^{\prime}, \alpha^{\prime}\right) \rightarrow\left(A^{\prime}, \alpha^{\prime}\right)$ in $\left(\mathbf{C} \downarrow B^{\prime}\right)$ is a pair $(\varphi, f)$, in which $\varphi=\left(\varphi_{0}, \varphi_{1}\right): G \rightarrow G^{\prime}$ is an internal functor and $f: A \rightarrow A^{\prime}$ a morphism in $\mathbf{C}$, making the standard diagram

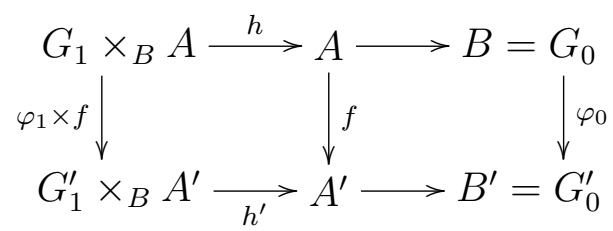


commute. On the object level Tors $\mathbf{C}$ coincides with the disjoint union of the categories $\operatorname{Tors}_{\mathbf{C}}(B, G)$ of $G$-torsors in $(\mathbf{C} \downarrow B)$, for all objects $B$ in $\mathbf{C}$ and all groups $G$ in $(\mathbf{C} \downarrow B)$. However this disjoint union has less morphisms of course: in fact a morphism $(\varphi, f)$ in Tors $_{\mathbf{C}}$ between two objects in the same $\operatorname{Tors}_{\mathbf{C}}(B, G)$ belongs to $\operatorname{Tors}_{\mathbf{C}}(B, G)$ if and only if $\varphi=1_{G}$.

Definition 2.2. (a) An extension in a pointed finitely complete category $\mathbf{C}$ is a diagram in $\mathbf{C}$ of the form

$$
K \stackrel{\varkappa}{\longrightarrow} A \stackrel{\alpha}{\longrightarrow} B
$$

where $\alpha$ is an effective descent morphism, and $\varkappa$ is a kernel of $\alpha$. Assuming $B$ and $K$ fixed, we will also say that $(A, \alpha, \varkappa)$ is an extension of $B$ with kernel $K$, and the category of such extensions will be denoted by $\operatorname{Ext}_{\mathbf{C}}(B, K)$.

(b) A splitting for an extension above is a morphism $\beta: B \rightarrow A$ with $\alpha \beta=1$; a split extension is an extension equipped with a specified splitting, and therefore morphisms of split extensions are supposed to commute with splittings.

Consider the functor

$$
\Phi_{B, G, K, \iota}: \operatorname{Tors}_{\mathbf{C}}(B, G) \longrightarrow \operatorname{Ext}_{\mathbf{C}}(B, K),
$$

constructed as follows:

- we begin with an arbitrary object $B$ in a pointed finitely complete category $\mathrm{C}$;

- then take $G$ to be a group in $(\mathbf{C} \downarrow B)$, and its underlying object in $(\mathbf{C} \downarrow B)$ will be written as $\left(G_{1}, d\right)$;

- we will take $K$, equipped with a fixed morphism $\iota: K \rightarrow G_{1}$, to be a fixed kernel of $d$;

- for every $G$-torsor $((A, \alpha), h)$, the morphism $\alpha$ has the same kernel $K$, more precisely its kernel can be presented as the composite $\varkappa=$

$$
K \stackrel{\langle\iota, 0\rangle}{\longrightarrow} G_{1} \times{ }_{B} A \stackrel{h}{\longrightarrow} A,
$$

and we put $\Phi_{B, G, K, \iota}((A, \alpha), h)=(A, \alpha, \varkappa)$.

Theorem 2.3. If the ground category $\mathbf{C}$ is (pointed and) protomodular, then the functor $\Phi_{B, G, K, \iota}$ above is full and faithful.

Proof. We have to prove that if $((A, \alpha), h)$ and $\left(\left(A^{\prime}, \alpha^{\prime}\right), h^{\prime}\right)$ are $G$-torsors and $f: A \rightarrow A^{\prime}$ a morphism making the diagram

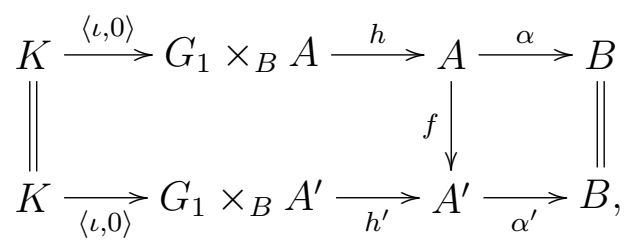


commute, then the diagram

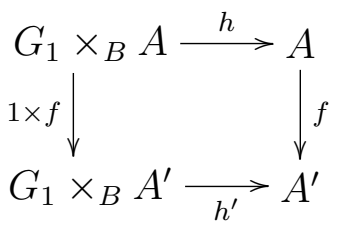

also commutes. For, consider the diagram

$$
K \stackrel{\langle\iota, 0\rangle}{\longrightarrow} G_{1} \times_{B} A \underset{\langle e \alpha, 1\rangle}{\stackrel{\mathrm{pr}_{2}}{\rightleftarrows}} A,
$$

where $e: B \rightarrow G_{1}$ is the unit of the group $G$. Since $\langle\iota, 0\rangle$ is a kernel of the second projection $\mathrm{pr}_{2}: G_{1} \times_{B} A \rightarrow A$ and $\langle e \alpha, 1\rangle$ splits this projection, the morphisms $\langle\iota, 0\rangle$ and $\left\langle e \alpha, 1_{A}\right\rangle$ are jointly epic by protomodularity [4, Proposition 11]. Since we also have

$$
\begin{aligned}
f h\langle\iota, 0\rangle & =h^{\prime}\langle\iota, 0\rangle=h^{\prime}(1 \times f)\langle\iota, 0\rangle \text { and } \\
f h\langle e \alpha, 1\rangle & =f=h^{\prime}\left\langle e \alpha^{\prime}, 1\right\rangle f=h^{\prime}\left\langle e \alpha^{\prime} f, f\right\rangle=h^{\prime}(1 \times f)\langle e \alpha, 1\rangle,
\end{aligned}
$$

we conclude that $f h=h^{\prime}(1 \times f)$, as desired.

Now consider the same construction but with variable $B$ and $G$, which is nothing but the forgetful functor

$$
\Phi: \operatorname{Tors}_{\mathbf{C}} \longrightarrow \operatorname{Ext}_{\mathbf{C}}, \quad \Phi((A, \alpha), h)=(A, \alpha)
$$

from Tors $_{\mathbf{C}}$ to the category $\mathbf{E x t}_{\mathbf{C}}$ of effective descent morphisms in C. Again, on the object level $\mathbf{E x t}_{\mathbf{C}}$ can be identified with the union (which is "almost" disjoint!) of all $\operatorname{Ext}_{\mathbf{C}}(B, K)$, but this union has less morphisms of course.

Theorem 2.4. If the ground category $\mathbf{C}$ is a (pointed) Mal'tsev category, then the functor $\Phi$ above is full and faithful, and an effective descent morphism $A \rightarrow$ $B$ is contained in its image if and only if it satisfies the equivalent conditions of 1.1 .

Proof. The functor $\Phi$ is nothing but the composite of the following two functors:

(i) the category equivalence

$$
\operatorname{Tors}_{\mathbf{C}} \sim \mathrm{REP}_{\mathbf{C}}
$$

where $\mathbf{R E P}_{\mathbf{C}}$ is the category of regular endo-pregroupoids in $\mathbf{C}$ (see the end of Section 1; the equivalence easily follows from the results of A. Kock [17], [18]);

(ii) the full and faithful forgetful functor from $\mathbf{R E P}_{\mathbf{C}}$ to $\mathbf{E x t}_{C}$ (see Theorem $1.2)$.

Note that one could deduce Theorem 2.3 from Theorem 2.4, but the direct proof we gave is much simpler of course; on the other hand, for a protomodular C, one could also deduce 2.4 from 2.3. Furthermore, although each $\Phi_{B, G, K, \iota}$ can be considered as a restriction of $\Phi$, one more theorem is needed to see the relationship between all these functors. 
Theorem 2.5. Let $\Phi_{B, G, K, \iota}$ be as in Theorem 2.3 and $\Phi_{B, G, K, \iota^{\prime}}$ another similar functor with the same $B$ and $K$. Then the following conditions are equivalent:

(a) the functors $\Phi_{B, G, K, \iota}$ and $\Phi_{B, G, K, \iota^{\prime}}$ have the same images (in $\operatorname{Ext}_{\mathbf{C}}(B, K)$ );

(b) the images of the functors $\Phi_{B, G, K, \iota}$ and $\Phi_{B, G, K, \iota^{\prime}}$ have a nonempty intersection;

(c) the category $\operatorname{Ext}_{\mathbf{C}}(B, K)$ has a connected component that has nonempty intersections with each of the images of $\Phi_{B, G, K, \iota}$ and $\Phi_{B, G, K, \iota^{\prime}}$;

(d) there exists an (iso) morphism $\theta=$

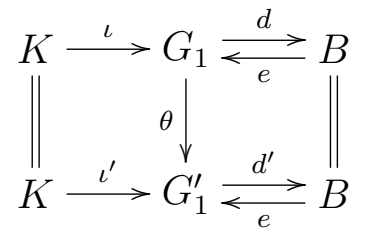

of split extensions.

Proof. Since the implications $(\mathrm{a}) \Rightarrow(\mathrm{b}) \Rightarrow(\mathrm{c})$ are trivial, it suffices to prove $(\mathrm{c}) \Rightarrow(\mathrm{d})$ and $(\mathrm{d}) \Rightarrow(\mathrm{a})$.

$(c) \Rightarrow(d)$ : As follows from protomodularity and the results of [10], the category $\operatorname{Ext}_{\mathbf{C}}(B, K)$ is a groupoid. Therefore the condition (c) means that there exist a $G$-torsor $((A, \alpha), h)$, a $G^{\prime}$-torsor $\left(\left(A^{\prime}, \alpha^{\prime}\right), h^{\prime}\right)$, and an isomorphism $f=$

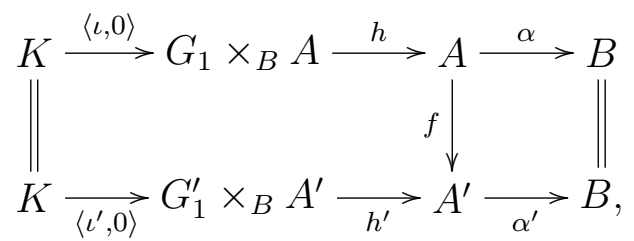

in $\mathbf{E x t}_{\mathbf{C}}(B, K)$. Since $f$ is also an isomorphism in $\mathbf{E x t}_{\mathbf{C}}$, Theorem 2.4 tells us that there exists an isomorphism $\varphi=\left(\varphi_{0}, \varphi_{1}\right): G \rightarrow G^{\prime}$ such that the pair $(\varphi, f)$ is an isomorphism from the torsor $h: G \times(A, \alpha) \rightarrow(A, \alpha)$ to the torsor $h^{\prime}: G^{\prime} \times\left(A^{\prime}, \alpha^{\prime}\right) \rightarrow\left(A^{\prime}, \alpha^{\prime}\right)$. Moreover, $\varphi_{0}=1$ according to the construction of $\Phi$ in Theorem 2.4. After that we take $\theta=\varphi_{1}$ and all we will have to prove is that $\varphi_{1} \iota=\iota^{\prime}$. We observe:

(i) Since $(\varphi, f)$ is a morphism of torsors, we have $h^{\prime}\left(\varphi_{1} \times f\right)=f h$.

(ii) Since the (left-hand square of the) diagram above commutes, (i) implies $h^{\prime}\left(\varphi_{1} \times f\right)\langle\iota, 0\rangle=h^{\prime}\left\langle\iota^{\prime}, 0\right\rangle$.

(iii) For the second projection $\operatorname{pr}_{2}: G_{1}^{\prime} \times_{B} A^{\prime} \rightarrow A^{\prime}$, we also have $\operatorname{pr}_{2}\left(\varphi_{1} \times f\right)$ $\langle\iota, 0\rangle=\operatorname{pr}_{2}\left\langle\iota^{\prime}, 0\right\rangle$.

(iv) Since $\left(A^{\prime}, \alpha^{\prime}\right)$ is a $G^{\prime}$-torsor, $h^{\prime}$ and $\operatorname{pr}_{2}$ are jointly monic, we conclude $\left(\varphi_{1} \times f\right)\langle\iota, 0\rangle=\left\langle\iota^{\prime}, 0\right\rangle$, and then $\varphi_{1} \iota=\operatorname{pr}_{1}\left(\varphi_{1} \times f\right)\langle\iota, 0\rangle=\operatorname{pr}_{1}\left\langle\iota^{\prime}, 0\right\rangle=\iota^{\prime}$, as desired.

$(\mathrm{d}) \Rightarrow(\mathrm{a})$ : Suppose $(\mathrm{d})$ holds, and $\left((A, \alpha), h^{\prime}\right)$ is a $G^{\prime}$-torsor. We observe:

(i) It is well known and follows, for example, from [12, Proposition 3.5], that a pointed object in a Mal'tsev category admits at most one group structure. Since $\theta$ is an isomorphism from the underlying pointed object of $G$ to the underlying pointed object of $G^{\prime}$, we conclude that it is a group isomorphism. 
(ii) As follows from (i), there exists a unique $G$-torsor structure $h: G \times$ $(A, \alpha) \rightarrow(A, \alpha)$ such that $((\theta, 1), 1)$ is a torsor isomorphism from $h: G \times$ $(A, \alpha) \rightarrow(A, \alpha)$ to $h^{\prime}: G^{\prime} \times(A, \alpha) \rightarrow(A, \alpha)$.

(iii) Since $((\theta, 1), 1)$ is a torsor isomorphism, $h\langle\iota, 0\rangle=h^{\prime}(\theta \times 1)\langle\iota, 0\rangle=h^{\prime}\left\langle\iota^{\prime}, 0\right\rangle$. Therefore $\Phi_{B, G, K, \iota}((A, \alpha), h)=\Phi_{B, G, K, \iota^{\prime}}\left((A, \alpha), h^{\prime}\right)$.

Since both $\Phi_{B, G, K, \iota}$ and $\Phi_{B, G, K, \iota^{\prime}}$ are fully faithful, and since the condition (d) is symmetric with respect to them, this means that they have the same images, as desired.

\section{The Functor Opext}

In this section we assume the ground category $\mathbf{C}$ to be a pointed protomodular category with semidirect products in the sense of [10]; in particular it could be any semi-abelian category. We will write $\operatorname{Tors}_{\mathbf{C}}(B, G)$ and $\operatorname{Ext}_{\mathbf{C}}(B, K)$ for the sets of isomorphism classes of objects in $\operatorname{Tors}_{\mathbf{C}}(B, G)$ and $\operatorname{Ext}_{\mathbf{C}}(B, K)$ respectively, and freely use the notation from Section 2.

Let Ker : $\operatorname{Pt}(B) \rightarrow \mathbf{C}$ be the functor from the category $\operatorname{Pt}(B)$ of triples $(A, \alpha, \beta)$, consisting of a split epimorphism $\alpha: A \rightarrow B$ and a splitting $\beta: B \rightarrow A$ of it, to $\mathbf{C}$, sending an $(A, \alpha, \beta)$ to a specified kernel of $\alpha$. We recall from [10] that this functor is monadic, and we will write $T^{B}$ for the corresponding monad on C. $T^{B}$-algebras will be called $B$-objects, and $T^{B}$-algebra structures will be called a $B$-actions, as in [3], where these actions were studied systematically. Let us also recall that a semidirect product $B \ltimes(X, \xi)$ of the object $B$ and a $B$-algebra $(X, \xi)$ is defined in [10] as the object in $\operatorname{Pt}(B)$ corresponding to $(X, \xi)$ under the canonical equivalence between $\operatorname{Pt}(B)$ and the category $\mathbf{C}^{B}$ of $T^{B}$-algebras $\left(=B\right.$-objects). If $(X, \xi)$ was a group in $\mathbf{C}^{B}$, then $B \ltimes(X, \xi)$ is a group in $\operatorname{Pt}(B)$, and hence also a group in $(\mathbf{C} \downarrow B)$. Let us recall again that a group in a pointed Mal'tsev category is nothing but an abelian object.

We define the functor

$$
\operatorname{Opext}_{\mathbf{C}}(B,-):\left[\text { Abelian objects in } \mathbf{C}^{B}\right] \longrightarrow[\text { Abelian groups }]
$$

as follows:

We take Opext $\operatorname{Ope}_{\mathbf{C}}(B,(X, \xi))=\operatorname{Tors}_{\mathbf{C}}(B, B \ltimes(X, \xi))$, which makes $\operatorname{Opext}_{\mathbf{C}}(B,-)$ a functor into the category of sets. General theory of torsors also tells us that this functor preserves (finite) products, and so sends groups to groups. And then we observe that since every abelian object in a pointed Mal'tsev category is also an abelian group in the category of its abelian objects, such a functor can also be considered as a functor into the category of abelian groups.

From the results of Section 2 we obtain:

Theorem 3.1. For every object $B$ and every abelian object $K$ in $\mathbf{C}$, the canonical map

$$
\bigsqcup_{\xi \in \Xi} \operatorname{Opext}(B,(K, \xi)) \longrightarrow \operatorname{Ext}(B, K)
$$

where $\Xi$ is the set of all $B$-actions on $K$ that make $(K, \xi)$ an abelian object in $\mathbf{C}^{B}$, is injective, and an extension $K \rightarrow A \rightarrow B$ is in its image if and only if 
$A \rightarrow B$ satisfies the equivalent conditions of 1.1. In particular that canonical map is bijective whenever $\mathbf{C}$ is strongly protomodular (in the sense of [7]).

Remark 3.1. (a) Originally we followed Gerstenhaber and described the image above as consisting of all extensions $K \rightarrow A \rightarrow B$ in which the induced monomorphism $K \rightarrow A \times_{B} A$ is normal, but then the first author has shown [5] that it is the same condition an in the present Theorem 3.1 above.

(b) As follows from the results of [11], the varieties of universal algebras to which Theorem 3.1 applies are precisely the BIT speciale varieties in the sense of A. Ursini [26] that are pointed. Much weaker but still a very useful description of extensions satisfying 1.1 in an arbitrary Mal'tsev variety was obtained by J. D. H. Smith [25]. As essentially follows from our Theorem 2.4, this result certainly extends to Mal'tsev categories.

\section{REFERENCES}

1. M. BARR, A survey of homology theories. Invited Talk on Workshop on Categorical Structures for Descent and Galois Theory, Hopf Algebras and Semiabelian Categories, Fields Institute, Toronto, 2002.

2. F. Borceux and D. Bourn, Mal'cev, protomodular, homological and semi-abelian categories. Mathematics and its Applications, 566. Kluwer Academic Publishers, Dordrecht, 2004.

3. F. Borceux, G. Janelidze, and G. M. Kelly, Internal object actions (sumitted).

4. D. Bourn, Normalization equivalence, kernel equivalence and affine categories. Category theory (Como, 1990), 43-62, Lecture Notes in Math., 1488, Springer, Berlin, 1991.

5. D. Bourn, Normal subobjects and abelian objects in protomodular categories. J. Algebra 228(2000), No. 1, 143-164.

6. D. Bourn, Commutator theory in regular Mal'tsev categories. Fields Institute Communication Series 43(2004) (to appear).

7. D. Bourn, Commutator theory in strongly protomodular categories. Univ. Littoral, Preprint 208, 2004.

8. D. Bourn and M. Gran, Centrality and connectors in Maltsev categories. Algebra Universalis 48(2002), No. 3, 309-331.

9. D. Bourn and M. Gran, Centrality and normality in protomodular categories. CT2000 Conference (Como). Theory Appl. Categ. 9(2001/02), 151-165.

10. D. Bourn and G. Janelidze, Protomodularity, descent, and semidirect products. Theory Appl. Categ. 4(1998), No. 2, 37-46.

11. D. Bourn and G. JANELIDze, Characterization of protomodular varieties of universal algebras. Theory Appl. Categ. 11(2003), No. 6, 143-147.

12. A. Carboni, M. C. Pedicchio, and N. Pirovano, Internal graphs and internal groupoids in Mal'cev categories. Category theory 1991 (Montreal, PQ, 1991), 97-109, CMS Conf. Proc., 13, Amer. Math. Soc., Providence, RI, 1992.

13. M. Gerstenhaber, A categorical setting for the Baer extension theory. Applications of Categorical Algebra (Proc. Sympos. Pure Math., Vol. XVII, New York, 1968), 50-64. Amer. Math. Soc., Providence, R.I., 1970.

14. P. J. Higgins, Groups with multiple operators. Proc. London Math. Soc. (3) 6(1956), $366-416$. 
15. G. Janelidze and M. C. Pedicchio, Pseudogroupoids and commutators. Theory Appl. Categ. 8(2001), No. 15, 408-456.

16. G. Janelidze, L. Márki, and W. Tholen, Semiabelian categories. J. Pure Appl. Algebra 168(2002), No. 2-3, 367-386.

17. A. Kock, Fibre bundles in general categories. J. Pure Appl. Algebra 56(1989), No. 3, $233-245$.

18. A. Kock, Generalized fibre bundles. Categorical algebra and its applications (LouvainLa-Neuve, 1987), 194-207, Lecture Notes in Math., 1348, Springer, Berlin, 1988.

19. S. Mac Lane, Homology. Die Grundlehren der mathematischen Wissenschaften, Bd. 114. Academic Press, Inc., Publishers, New York; Springer-Verlag, Berlin-GttingenHeidelberg, 1963.

20. G. Orzech, Obstruction theory in algebraic categories. I. J. Pure Appl. Algebra 2(1972), 287-314.

21. M. C. Pedicchio, A categorical approach to commutator theory. J. Algebra 177(1995), No. 3, 647-657.

22. M. C. Pedicchio, Arithmetical categories and commutator theory. The European Colloquium of Category Theory (Tours, 1994). Appl. Categ. Structures 4(1996), No. 2-3, 297-305.

23. M. C. Pedicchio, Some remarks on internal pregroupoids in varieties. Comm. Algebra 26(1998), No. 6, 1737-1744.

24. D. Rodelo, Moore categories. Theory Appl. Categ. 12(2004), No. 6, 237-247.

25. J. D. H. Sмith, Mal'tsev varieties. Lecture Notes in Mathematics 554, Springer, 1976.

26. A. Ursini, Osservazioni sulle varietá BIT. Boll. Un. Mat. Ital. (4) 7(1973), 205-211.

(Received 10.06.2004)

Authors' addresses:

Dominique Bourn

Université du Littoral, Calais

France

E-mail: bourn@lmpa.univ-littoral.fr

George Janelidze

A. Razmadze Mathematical Institute

Georgian Academy of Sciences

1, M. Aleksidze St., Tbilisi 0193

Georgia

E-mail: gjanel@rmi.acnet.ge 